\title{
Parameterized complexity of machine scheduling: 15 open problems
}

\author{
Matthias Mnich ${ }^{\mathrm{a}, \mathrm{b}}$, René van Bevern ${ }^{\mathrm{c}, \mathrm{d}, *}$ \\ ${ }^{a}$ Universität Bonn, Bonn, Germany \\ ${ }^{b}$ Maastricht University, Maastricht, The Netherlands \\ ${ }^{c}$ Department of Mechanics and Mathematics, Novosibirsk State University, Novosibirsk, Russian Federation \\ ${ }^{d}$ Sobolev Institute of Mathematics of the Siberian Branch of the Russian Academy of Sciences, Novosibirsk, Russian Federation
}

\begin{abstract}
Machine scheduling problems are a long-time key domain of algorithms and complexity research. A novel approach to machine scheduling problems are fixed-parameter algorithms. To stimulate this thriving research direction, we propose 15 open questions in this area whose resolution we expect to lead to the discovery of new approaches and techniques both in scheduling and parameterized complexity theory.
\end{abstract}

Keywords: parallel machines, shop scheduling, makespan, total completion time, total tardiness, throughput, number of tardy jobs

\section{Introduction}

Algorithms for machine scheduling problems form one of the core applications of combinatorial optimization. In those problems, we are generally given a finite set $J$ of jobs with certain characteristics, and we must find a schedule for processing the jobs on one or more machines, which also may have their individual specifications. Typical characteristics of a job $j$ are its processing time $p_{j} \in \mathbb{N}$, its release date $r_{j} \in \mathbb{N}$, its due date $d_{j} \in \mathbb{N}$, or its importance reflected by a weight $w_{j} \in \mathbb{N}$. Jobs may be subject to precedence constraints enforcing some jobs to be completed before other jobs start. Also, jobs may be preempted, or may be required to be processed without preemption. Machine characteristics typically include their speed or whether they are capable of processing a certain type of job.

Usually, one is not only searching for a feasible schedule that respects all constraints, but additionally optimizes some objective function. Classical objectives include the minimization of the makespan or the sum of weighted completion times (which is equivalent to minimizing the weighted average completion time). Since the inception of the field in the 1950s, thousands of research papers have been devoted to understanding the complexity of scheduling problems. A significant portion of investigated problems turned out to be NP-hard. In consequence, algorithm designers proposed algorithms for these problems that yield approximate solutions in polynomial time. In 1999, Schuurman and Woeginger (1999) listed 10 of the most prominent open problems around polynomial-time approximation algorithms for NP-hard scheduling problems at that time.

Only recently, a different algorithmic approach has been put forward for solving NP-hard scheduling problems: fixed-param-

${ }^{*}$ Corresponding author. René van Bevern, Department of Mechanics and Mathematics, Novosibirsk State University, ul. Pirogova 2, 630090 Novosibirsk, Russian Federation

Email addresses: mmnich@uni-bonn.de (Matthias Mnich), rvb@nsu.ru (René van Bevern) eter algorithms. The idea in fixed-parameter algorithms is to accept exponential running times, which are seemingly inevitable in solving NP-hard problems, but to restrict them to certain aspects of the problem, which are captured by parameters (Cygan et al., 2015; Flum and Grohe, 2006; Niedermeier, 2006). More formally, fixed-parameter algorithms solve an instance of size $n$ with parameter $k$ in $f(k) \cdot \operatorname{poly}(n)$ time for some computable, typically superpolynomial, function $f$. Thus, fixed-parameter algorithms can solve even large instances of NP-hard scheduling problems if the parameter takes only small values, the function $f$ grows only moderately, and the polynomial degree in $n$ is small (van Bevern et al., 2015). Moreover, problems that are even difficult to approximate can be approximated efficiently and well in real-world instances using fixed-parameter approximation algorithms that exploit small parameters of real-world data (van Bevern et al., 2017a).

While fixed-parameter algorithms are now a well-investigated area of algorithmics, their systematic application to scheduling problems has gained momentum only recently (van Bevern and Pyatkin, 2016; Chen et al., 2017; Cieliebak et al., 2004; Halldórsson and Karlsson, 2006; Hermelin et al., 2015, 2018; Jansen et al., 2017; Marx and Schlotter, 2011, and more references below). This already led to the transfer of proof techniques from parameterized complexity to the world of scheduling, such as $n$-fold integer programming (Knop and Koutecký, 2017), color coding, problem kernelization (van Bevern et al., 2015), and W-hardness (Bentert et al., 2017; Bodlaender and Fellows, 1995; van Bevern et al., 2016, 2017b; Mnich and Wiese, 2015; Fellows and McCartin, 2003; Hermelin et al., 2017). It also led to the transfer of techniques from mathematical programming to parameterized complexity, such as convex integer programming (Mnich and Wiese, 2015) or parameterizing by structural properties of the integer feasible polytope of linear programs (Jansen and Klein, 2017).

In the following, we summarize known results and list open 
problems regarding the parameterized complexity of scheduling problems on a single machine, on parallel identical machines, and in shop scheduling environments. We do not claim these problems to be the most important ones, but expect that their resolution (in one way or the other) will lead to the discovery of further new approaches both in parameterized complexity and scheduling theory, thus stimulating further research with both practical and theoretical significance.

In this sense, we hope that this work will be appealing and inspiring both to researchers with a scheduling background, as well as to researchers with a parameterized complexity background. For the latter, we exhibit some fixed-parameter tractability results that appeared before the advent of parameterized complexity and thus were not explicitly described as such.

Organization of this work. We start by giving preliminaries about machine scheduling and parameterized complexity in Section 2. Then we look at single-machine problems in Section 3, and parallel identical machines in Section 4. We finally consider the broad class of shop scheduling problems in Section 5.

\section{Preliminaries}

This section defines basic notions of scheduling theory, parameterized complexity theory, and approximation algorithms.

\subsection{Scheduling theory}

Throughout this work, we use the standard three-field notation of scheduling problems due to Graham et al. (1979). This allows us to denote many problems as a triple $\alpha|\beta| \gamma$, where $\alpha$ is the machine environment, $\beta$ are job characteristics and scheduling constraints, and $\gamma$ is the objective function.

\subsubsection{Machine models}

We consider the following machine environments $\alpha$, which are described in detail in the following. Single-machine environments are denoted by the symbol 1, parallel identical machines are denoted by $P$, job shop, open shop, and flow shop environments are denoted by $J, O$, and $F$, respectively.

The symbol describing the machine environment can be followed by an integer restricting the number of machines (for example, $P 2$ indicates two parallel identical machines). If the symbol is followed by $m$ (for example, $P m$ ), this indicates that the number of machines is an arbitrary constant. If the symbol is neither followed by a number nor $m$, then the number of machines is assumed to be given as part of the input.

Single-machine environments (denoted by " 1 " in the $\alpha$-field). Each job $j$ has to be processed for a given amount $p_{j} \in \mathbb{N}$ of time (its processing time) on a single machine. The machine can process only one job at a time.

Parallel identical machines (denoted by " $P$ " in the $\alpha$-field). We are given a number $m$ of parallel identical machines (that is, of the same speed). Each job $j$ has to be processed by exactly one machine for a given amount $p_{j} \in \mathbb{N}$ of time and each machine can process only one job at a time.
Unrelated parallel machines (denoted by " $R$ " in the $\alpha$-field). We are given a number $m$ of machines. Each job $j$ has to be processed by exactly one machine $i$ for a given amount $p_{i j} \in \mathbb{N}$ of time and each machine can process only one job at a time.

Shop scheduling problems. In shop scheduling problems, we are given a set $M$ of machines and a set $J$ of $n$ jobs, where each job $j$ consists of $n_{j} \in \mathbb{N}$ operations. In the most general setting, the operations of each job are partially ordered: an operation of a job can only start once its preceding operations are completed. Processing an operation $o_{i j}$ of job $j$, where $i \in\left\{1, \ldots, n_{j}\right\}$, requires time $p_{i j}$ on a certain machine $\mu_{i j}$. Each job can be processed by at most one machine at a time and each machine can process at most one operation at a time. The three most important classes of shop scheduling problems are:

Open shop scheduling, denoted by "O" in the $\alpha$-field: the processing order of the operations of each job is unrestricted and must be decided by an algorithm. The only constraint is that each job has exactly one operation on each machine.

Job shop scheduling, denoted by "J" in the $\alpha$-field: the operations of each job are totally ordered, yet each job may have a distinct total order on its operations. Herein, several operations may require processing on the same machine and not every job may have operations on all machines.

Flow shop scheduling, denoted by " $\mathrm{F}$ " in the $\alpha$-field: all jobs have the same set of operations with the same total order.

\subsubsection{Job characteristics}

For job characteristics, we add qualifiers to the $\beta$-field. In this survey, we consider the following types of job characteristics.

Precedence constraints. If jobs are restricted by precedence constraints, then we add the qualifier "prec" to the $\beta$-field to indicate that jobs are only allowed to start after their predecessors are completed. In the case where the partial order induced by the precedence constraints is the disjoint union of total orders, we write "chains" instead of "prec".

Processing time restrictions. If all jobs $j$ have unit processing time, we add " $p_{j}=1$ " to the $\beta$-field. If all jobs $j$ have an equal processing time $p$, we add " $p_{j}=p$ " to the $\beta$-field. In case where processing times are bounded from above by some constant $c$, we add " $p_{j} \leq c$ " to the $\beta$-field. If the processing times are restricted to values in some set $P \subseteq \mathbb{N}$, we add " $p_{j} \in P$ " to the $\beta$-field.

Machine restrictions. If each job $j$ can be processed only on a subset $M_{j}$ of machines, we add " $M_{j}$ " to the $\beta$-field.

Release dates. Each job $j$ may have a release date $r_{j}$, before which it cannot be started. In this case, we add " $r_{j}$ " to the $\beta$-field.

Preemption. Per default, we assume that jobs are not allowed to be preempted. Otherwise, we add "pmtn" to the $\beta$-field.

Multiprocessor jobs. When a job $j$ may occupy size ${ }_{j}$ machines during execution, we add the qualifier "size ${ }_{j}$ " to the $\beta$-field; such problems are known as "multiprocessor scheduling". If the number of required machines is restricted to values in some set $S \subseteq \mathbb{N}$, we add "size ${ }_{j} \in S$ " to the $\beta$-field. 
Batch setup times. When jobs are partitioned into batches and a sequence-dependent setup time $s_{p q}$ is needed when switching from a job of batch $p$ to a job of batch $q$, then, in accordance with Allahverdi et al. (2008), we add "ST ${ }_{\mathrm{sd}, b}$ " to the $\beta$-field.

\subsubsection{Objective functions}

The objective functions $\gamma$ that we aim to minimize are

- the makespan $C_{\max }=\max \left\{C_{j} \mid j \in J\right\}$, where $C_{j}$ is the completion time of job $j$ in a schedule, and

- the weighted sum of completion times, $\sum_{j \in J} w_{j} C_{j}$, when each job $j \in J$ also has a weight $w_{j} \in \mathbb{N}$.

If each job $j \in J$ has a due date $d_{j} \in \mathbb{N}$, we also minimize

- the total weighted tardiness $\sum_{j \in J} w_{j} T_{j}$, where

$$
T_{j}=\max \left\{0, d_{j}-C_{j}\right\}, \text { and }
$$

- the weighted number of tardy jobs, $\sum_{j \in J} w_{j} U_{j}$, where $U_{j}=0$ if job $j$ is finished by its due date and $U_{j}=1$ otherwise.

This is equivalent to maximizing throughput - the weighted number of jobs that get finished by their due dates.

Generally, we will drop the " $j \in J$ " subscript under the sum and we will refer to the unit-weight variants of the scheduling problems by simply dropping the $w_{j}$ from the objective functions.

\subsection{Parameterized complexity}

An instance of a parameterized problem $\Pi \subseteq \Sigma^{*} \times \mathbb{N}$ is a pair $(x, k)$ consisting of the input $x$ and the parameter $k$. A parameterized problem $\Pi$ is fixed-parameter tractable (FPT) if there is an algorithm solving any instance of $\Pi$ with parameter $k$ and size $n$ in $f(k) \cdot \operatorname{poly}(n)$ time for some computable function $f$. Such an algorithm is called a fixed-parameter algorithm.

Note that fixed-parameter tractability for a parameter $k$ is a much stronger property than polynomial-time solvability for constant $k$ : a fixed-parameter algorithm running in $O\left(2^{k} \cdot n\right)$ time takes polynomial time even for $k \in O(\log n)$. In contrast, an algorithm running in time $O\left(n^{k}\right)$ is polynomial for constant $k$, but is often impractical even for small values of $k$.

The main goal of parameterized complexity is determining which parameterized problems have fixed-parameter algorithms. These can efficiently solve NP-hard problems in applications where the parameter takes only small values and the degree of the polynomial in $n$ is small. Notably, a problem may be fixedparameter tractable with respect to one parameter, but might be not with respect to another. How to find parameters that are both small in applications and lead to fixed-parameter algorithms, is a research branch on its own (Fellows et al., 2013; Komusiewicz and Niedermeier, 2012; Niedermeier, 2010).
Problem kernelization. Parameterized complexity enabled a mathematical formalization of polynomial-time data reduction with provable performance guarantees: kernelization. It has been successfully applied to obtain effective polynomial-time data reduction algorithms for many NP-hard problems (Guo and Niedermeier, 2007; Kratsch, 2014) and also led to techniques for proving lower bounds on the effectivity of polynomial-time data reduction (Misra et al., 2011; Bodlaender et al., 2014).

A kernelization algorithm for a parameterized problem $\Pi \subseteq$ $\Sigma^{*} \times \mathbb{N}$ reduces an instance $(x, k)$ to an instance $\left(x^{\prime}, k^{\prime}\right)$ in polynomial time such that the size of $x^{\prime}$ and $k^{\prime}$ depends only on $k$ and such that $(x, k) \in \Pi$ if and only if $\left(x^{\prime}, k^{\prime}\right) \in \Pi$. The instance $\left(x^{\prime}, k^{\prime}\right)$ is called a problem kernel.

Note that it is the parameter that allows us to measure the effectivity of polynomial-time data reduction since an absolute statement like "the data reduction shrinks the input by at least one bit" for an NP-hard problem would imply that we can solve NP-hard problems in polynomial time.

Parameterized intractability. To show that a problem is presumably not fixed-parameter tractable, there is a parameterized analogue of NP-hardness. The parameterized analogue of $\mathrm{P} \subseteq \mathrm{NP}$ is a hierarchy of complexity classes FPT $\subseteq \mathrm{W}[1] \subseteq \mathrm{W}[2] \subseteq$ $\cdots \subseteq \mathrm{XP}$, where $\mathrm{XP}$ is the class of parameterized problems that are solvable in polynomial time for constant parameter values. The working hypothesis is that all inclusions in this hierarchy are proper, paralleling the famous $\mathrm{P} \neq \mathrm{NP}$ conjecture.

A parameterized problem $\Pi$ is (weakly) W[t]-hard for some $t \in \mathbb{N}$ if any problem in $\mathrm{W}[t]$ has a parameterized reduction to $\Pi$ : a parameterized reduction from a parameterized problem $\Pi_{1}$ to a parameterized problem $\Pi_{2}$ is an algorithm mapping an instance $(x, k)$ to an instance $\left(x^{\prime}, k^{\prime}\right)$ in time $f(k) \cdot$ poly $(|x|)$ such that $k^{\prime} \leq g(k)$ and $(x, k) \in \Pi_{1} \Longleftrightarrow\left(x^{\prime}, k^{\prime}\right) \in \Pi_{2}$, where $f$ and $g$ are arbitrary computable functions. By definition, no $\mathrm{W}[t]$-hard problem is fixed-parameter tractable unless FPT $=\mathrm{W}[t]$.

A parameterized problem is strongly $W[1]$-hard if it is $\mathrm{W}[1]$ hard even if all numbers in the input are encoded in unary.

\subsection{Approximation algorithms}

Since we only consider minimization problems, we introduce approximation terminology only for minimization problems.

An $\alpha$-approximation is a solution to an optimization problem with cost at most $\alpha$ times the cost of an optimal solution. A polynomial-time approximation scheme (PTAS) is an algorithm that computes a $(1+\varepsilon)$-approximation in polynomial time for any constant $\varepsilon>0$. An efficient polynomial-time approximation scheme (EPTAS) is an algorithm that computes a $(1+\varepsilon)$-approximation in time $f(1 / \varepsilon) \cdot \operatorname{poly}(n)$ for some computable function $f$ and $\varepsilon>0$. Finally, a fully polynomial-time approximation scheme (FPTAS) is an algorithm that computes a $(1+\varepsilon)$-approximation in time $\operatorname{poly}(n, 1 / \varepsilon)$ for any $\varepsilon>0$.

It is known that any problem having an EPTAS is fixedparameter tractable parameterized by the cost of an optimal solution (Cesati and Trevisan, 1997, Lemma 11). Thus, showing W[1]-hardness of a problem parameterized by the optimum solution cost shows that it neither has an EPTAS nor an FPTAS unless FPT $=$ W[1]. Moreover, strongly NP-hard optimization 
problems with polynomially bounded objective functions do not have FPTASes unless P = NP (Garey and Johnson, 1979).

\section{Single-machine problems}

In this section, we consider various single-machine problems: Section 3.1 studies total weighted tardiness as objective function, Section 3.2 the total weighted completion time, Section 3.3 the weighted number of tardy jobs, and, finally, Section 3.4 considers a variant with forbidden start and end times of jobs.

\subsection{Total weighted tardiness}

The problem $1 \| \Sigma w_{j} T_{j}$ is strongly NP-hard (Lawler, 1977; Lenstra et al., 1977), yet has two easier special cases: $1\left|p_{j}=p\right| \sum w_{j} T_{j}$ is solvable in polynomial time via a reduction to the classical linear assignment problem, whereas $1 \| \Sigma T_{j}$ is solvable in pseudo-polynomial time (Lawler, 1977; Lenstra et al., 1977).

To be more exact, Lawler (1977)'s pseudo polynomial-time algorithm is a fixed-parameter algorithm for $1 \| \Sigma T_{j}$ parameterized by the maximum processing time $p_{\max }$ that also works for $1 \| \Sigma w_{j} T_{j}$ with agreeable processing times and weights, that is, $p_{i}<p_{j}$ implies $w_{i} \geq w_{j}$.

Although $1 \| \Sigma w_{j} T_{j}$ is strongly NP-hard and thus cannot have pseudo polynomial-time algorithms unless $\mathrm{P}=\mathrm{NP}$, it is interesting whether the fixed-parameter tractability result for $1 \| \Sigma w_{j} T_{j}$ with agreeable processing times and weights holds in general:

Open Problem 1. Is $1 \| \Sigma w_{j} T_{j}$ fixed-parameter tractable parameterized by the maximum processing time $p_{\max }$ ?

Also note that, given that $1\left|p_{j}=p\right| \sum w_{j} T_{j}$ is solvable in polynomial time, it is interesting to see whether $1 \| \Sigma w_{j} T_{j}$ is fixed-parameter tractable with respect to the number $\bar{p}$ of distinct processing times.

\subsection{Total weighted completion time}

Ambühl and Mastrolilli (2009) showed that $1|\operatorname{prec}| \sum w_{j} C_{j}$ is a special case of Weighted Vertex Cover. Vertex Cover is one of the most well-studied problems in parameterized complexity theory, in particular in terms of problem kernels; small kernels for Weighted Vertex Cover were established by Etscheid et al. (2017). It is interesting which kernelization algorithms carry over to $1|\operatorname{prec}| \Sigma w_{j} C_{j}$. However, a problem kernel for $1 \mid$ prec $\mid \sum w_{j} C_{j}$ whose size is bounded by a function of the optimum is not interesting - the optimum is at least the weighted sum of all processing times and thus already bounds the input size without data reduction. Remarkably, VerTex Cover admits a (randomized) algorithm yielding a problem kernel with size polynomial in the difference between the optimum and a lower bound given by the relaxation of the natural ILP (Kratsch and Wahlström, 2012). Since $1 \mid$ prec $\mid \Sigma w_{j} C_{j}$ is a special case of Weighted Vertex Cover, and thus allows for a likewise natural ILP formulation (Ambühl and Mastrolilli, 2009), the following question is interesting:

Open Problem 2. Does $1 \mid$ prec $\mid \sum w_{j} C_{j}$ admit a problem kernel of size polynomial in the difference between the optimum and the lower bound obtained from its natural LP relaxation?
We point out that any partial progress on this question would be interesting: be it a randomized kernelization algorithm or even a partial kernel that bounds only the number of jobs and not necessarily their weights or processing times.

The question is complicated by the fact that each vertex in the Weighted Vertex Cover instance created by Ambühl and Mastrolilli (2009), and thus each variable in the corresponding ILP, corresponds to a pair of jobs in the $1|\operatorname{prec}| \sum w_{j} C_{j}$ instance, such that known data reduction rules for Weighted Vertex Cover do not allow for a straightforward interpretation in terms of jobs.

\subsection{Throughput or weighted number of tardy jobs}

In a survey on open questions in maximum throughput scheduling, Sgall (2012) asked whether there is a polynomial-time algorithm for $1\left|r_{j}, p_{j} \leq c\right| \Sigma U_{j}$ for constant $c$. Similarly, the NP-hardness of the weighted case for constant $c$ is open. Parameterized complexity can serve as an intermediate step towards resolving these questions.

Open Problem 3. Are $1\left|r_{j}\right| \Sigma U_{j}$ and $1\left|r_{j}\right| \Sigma w_{j} U_{j} W[1]$-hard parameterized by the maximum processing time $p_{\max }$ ?

Currently, even containment in the parameterized complexity class XP is open. The special case $1 \| \Sigma U_{j}$ is polynomial-time solvable, whereas $1 \| \Sigma w_{j} U_{j}$ is weakly NP-hard and solvable in pseudo-polynomial time (Lawler and Moore, 1969; Karp, 1972).

Hermelin et al. (2018) gave fixed-parameter algorithms for $1 \| \Sigma w_{j} U_{j}$ simultaneously parameterized by any two out of the following three parameters: the number of distinct due dates, the number of distinct processing times, and the number of distinct job weights.

Fellows and McCartin (2003) showed that $1 \mid$ prec, $p_{j}=1 \mid \Sigma U_{i}$ is W[1]-hard parameterized by the number of tardy jobs but fixed-parameter tractable with respect to this parameter if the partial order induced by the precedence constraints has constant width. Herein, the width of a partial order is the size of a largest set of mutually incomparable jobs.

\subsection{Forbidden start and end times}

Machine scheduling problems with forbidden start and end times model the situation when an additional resource, subject to unavailability constraints, is required to start or finish a job. For example, this resource might be operators of chemical experiments, which serves as a major motivation for such problems.

For makespan minimization on a single machine, Billaut and Sourd (2009) gave an algorithm that runs in $n^{O\left(\tau^{2}\right)}$ time for $\tau$ forbidden start times and $n$ jobs; this was improved by Rapine and Brauner (2013) to $n^{O(\tau)}$ time. For the high-multiplicity encoding of the input—given by binary numbers $n_{t}$ encoding the number of jobs having the same forbidden start and end timesGabay et al. (2016) showed a polynomial-time algorithm if the number $\tau$ of forbidden times is constant. All of these results leave open the possibility for fixed-parameter tractability of the problem parameterized by $\tau$.

Open Problem 4. Is makespan minimization on a single machine with $\tau$ forbidden start and end times fixed-parameter tractable parameterized by $\tau$ ? 


\section{Parallel identical machines}

In this section, we consider scheduling on parallel identical machines. Section 4.1 considers the makespan objective, Section 4.2 the makespan objective with precedence constraints, Section 4.3 the weighted number of tardy jobs, Section 4.4 considers just-in-time scheduling, and, finally, Section 4.5 considers variants with allowed preemption of jobs.

\subsection{Makespan}

Alon et al. (1998) showed an EPTAS for $P \| C_{\max }$, which implies that $P \| C_{\max }$ is fixed-parameter tractable parameterized by the makespan $C_{\max }$ (Cesati and Trevisan, 1997, Lemma 11).

This was improved by Mnich and Wiese (2015) to a fixedparameter algorithm for the maximum processing time $p_{\max }$ of any job; we generally expect $p_{\max } \ll C_{\max }$. The running time of both algorithms is doubly-exponential in the parameter. An improved algorithm whose running time depends singly-exponentially on $p_{\max }$ was proposed by Knop and Koutecký (2017). Very recently, Knop et al. (2017) strengthened this result by giving an algorithm with single-exponential dependence $p_{\max }$ even for the high-multiplicity encoding, when, for each processing time $p \leq p_{\max }$, the number of jobs with processing time $p$ is encoded in binary. Chen et al. (2017) generalized the result of Mnich and Wiese (2015) by showing that $R \| C_{\max }$ is fixedparameter tractable parameterized by $p_{\max }$ and the rank of the matrix $\left(p_{i j}\right)$ giving the processing time $p_{i j}$ of job $j$ on machine $i$.

In a breakthrough result, Goemans and Rothvoß (2014) showed that $P \| C_{\max }$ is polynomial-time solvable when the number $\bar{p}$ of distinct processing times is constant, that is, the problem is in XP. Their result holds even for the high-multiplicity encoding of the input, where the number $m$ of machines and the number $n_{j}$ of jobs with processing time $p_{j}$ are encoded in binary for each $j \in\{1, \ldots, \bar{p}\}$. A close inspection of their result reveals that it is a fixed-parameter algorithm when the processing times $p_{j}$ are encoded in unary. Despite all this progress, the following problem remains open.

Open Problem 5. Is $P \| C_{\max }$ with high-multiplicity encoding fixed-parameter tractable parameterized by the number $\bar{p}$ of distinct processing times, that is, when job processing times $p_{j}$ and the number of jobs for each processing time are encoded in binary?

To find a fixed-parameter algorithm for the high-multiplicity encoding of $P \| C_{\max }$ parameterized by $\bar{p}$, one difficulty is outputting an optimal schedule, whose obvious encoding requires at least $m$ bits (to store how many jobs of each processing time are processed on each machine) and is therefore neither polynomial in the input size nor bounded by a function of $\bar{p}$. Such problems might be possibly overcome by using the framework of Brauner et al. (2005).

\subsection{Makespan and precedence constraints}

The parameterized complexity of $P|\operatorname{prec}| C_{\max }$ and special cases has extensively been studied with respect to the width of the partial order induced by the precedence constraints: the width of a partial order is the size of its largest antichain-a set of pairwise incomparable jobs.

The special case $P 2 \mid$ chains $\mid C_{\max }$ is weakly NP-hard even for partial orders of width three (Günther et al., 2014; van Bevern et al., 2016). Since this excludes fixed-parameter algorithms using the partial order width as parameter already on two machines, it has been tried to use the partial order width and the maximum processing time as parameters simultaneously. Bodlaender and Fellows (1995) showed that even $P \mid$ prec, $p_{j}=1 \mid C_{\max }$ is $\mathrm{W}[2]$-hard parameterized by the partial order width and by the number of machines. Later, van Bevern et al. (2016) showed that combining partial order width with maximum processing time does not even yield fixed-parameter algorithms on two machines. More precisely, they showed that even $P 2 \mid$ prec, $p_{j} \in\{1,2\} \mid C_{\max }$ is $\mathrm{W}[2]$-hard parameterized by the partial order width; and so is $P 3 \mid$ prec, size $_{j} \in\{1,2\} \mid C_{\text {max }}$.

Further restricting this problem, one arrives at a long-standing open problem due to Garey and Johnson (1979, OPEN8) of whether $P 3 \mid$ prec, $p_{j}=1 \mid C_{\max }$ is NP-hard or polynomial-time solvable. In fact, the complexity is open for any constant number of machines. Thus, as pointed out by van Bevern et al. (2016), it would be surprising to show W[1]-hardness of this problem for any parameter, since this would exclude polynomial-time solvability unless FPT $=\mathrm{W}[1]$.

Open Problem 6. Is $P 3 \mid$ prec, $p_{j}=1 \mid C_{\max }$ fixed-parameter tractable parameterized by the width of the partial order induced by the precedence constraints?

Note that a negative answer would basically answer the open question of Garey and Johnson (1979) on whether the problem is polynomial-time solvable, whereas a positive answer would be in strong contrast to the $\mathrm{W}[2]$-hardness of the slight generalizations $P \mid$ prec, $p_{j}=1\left|C_{\max }, P 2\right|$ prec, $p_{j} \in\{1,2\} \mid C_{\max }$, and $P 3 \mid$ prec, size $_{j} \in\{1,2\} \mid C_{\max }$ considered by Bodlaender and Fellows (1995) and van Bevern et al. (2016).

\subsection{Throughput or weighted number of tardy jobs}

Baptiste et al. (2004) showed that $P m\left|r_{j}, p_{j}=p\right| \sum w_{j} U_{j}$ is polynomial-time solvable. According to Sgall (2012), this is open when the number of machines is not a constant. A first step to resolving this question is solving the following problem.

Open Problem 7. Are $P\left|r_{j}, p_{j}=p\right| \Sigma w_{j} U_{j}$ and $P\left|r_{j}, p_{j}=p\right| \Sigma U_{j}$ fixed-parameter tractable parameterized by the number of machines?

A negative answer to this question would also be interesting since it is open whether these problems are even NP-hard if the number $m$ of machines is part of the input. Notably, the special case $P\left|p_{j}=p\right| \sum w_{j} U_{j}$ is polynomial-time solvable via a reduction to the classical linear assignment problem but $P\left|p_{j}=p, \operatorname{pmtn}\right| \sum w_{j} U_{j}$ is strongly NP-hard (Brucker and Kravchenko, 1999).

\subsection{Interval scheduling or just-in-time scheduling}

An important special case of maximizing the throughput on parallel identical machines is interval scheduling, where each job $j$ has a weight $w_{j}$, a fixed start time, and a fixed end time. 
The goal is to schedule a maximum-weight subset of jobs nonpreemptively on $m$ parallel identical machines. As always, each machine can process only one job at a time. Since we can interpret this problem as minimizing the total weight of jobs not meeting their due dates, where each job $j$ has a processing time $p_{j}$, a release date $r_{j}$, and a due date $d_{j}$ such that $d_{j}-r_{j}=p_{j}$, we denote the problem as $P\left|d_{j}-r_{j}=p_{j}\right| \sum w_{j} U_{j}$. This problem is equivalent to maximizing the weighted number of "just-in-time" jobs on parallel identical machines (Sung and Vlach, 2005).

Arkin and Silverberg (1987) show that this problem is solvable in $O\left(n^{2} \log n\right)$ time. However, they showed that the variant $P\left|M_{j}, d_{j}-r_{j}=p_{j}\right| \sum w_{j} U_{j}$ is NP-hard and solvable in $O\left(n^{m+1}\right)$ time.

Open Problem 8. Is $P\left|M_{j}, d_{j}-r_{j}=p_{j}\right| \Sigma w_{j} U_{j}$ fixed-parameter tractable parameterized by the number of machines?

This problem is a special case of $R\left|d_{j}-r_{j}=p_{j}\right| \Sigma w_{j} U_{j}$ where each job $j$ has a processing time $p_{i j} \in\left\{p_{j}, \infty\right\}$ on machine $i$. In that sense, any positive answer to Open Problem 8 (in form of a fixed-parameter algorithm) can serve as a first step towards a fixed-parameter algorithm for $R\left|d_{j}-r_{j}=p_{j}\right| \sum w_{j} U_{j}$ also; after all, the $O\left(n^{m+1}\right)$-time algorithm of Arkin and Silverberg (1987) for $P\left|M_{j}, d_{j}-r_{j}=p_{j}\right| \sum w_{j} U_{j}$ was generalized to $R\left|d_{j}-r_{j}=p_{j}\right| \sum w_{j} U_{j}$ with essentially the same running time (Sung and Vlach, 2005).

We point out that, if we only slightly relax the condition $d_{j}-r_{j}=p_{j}$ to $d_{j}-r_{j} \leq \lambda p_{j}$ for any constant $\lambda>0$, then the problem is weakly NP-hard for $m=2$ and strongly W[1]hard parameterized by $m$ (van Bevern et al., 2017b), even when all jobs are allowed to be processed on all machines and only checking whether one can finish all jobs by their due date.

Using a construction due to Halldórsson and Karlsson (2006), $P\left|M_{j}, d_{j}-r_{j}=p_{j}\right| \sum w_{j} U_{j}$ can be seen to be a special case of the Job InTERVAl SElEction problem introduced by Nakajima and Hakimi (1982), where each job has multiple possible execution intervals and we have to select one execution interval for each job in order to process it: we model the machines by pairwise disjoint segments of the real line and each job has an interval in each segment belonging to a machine it can be processed on. Via this relation to Joв Interval Selection, one can model $P\left|M_{j}, d_{j}-r_{j}=p_{j}\right| \sum w_{j} U_{j}$ as Colorful IndePENDENT Set in an interval graph with at most $m n$ intervals colored in $n$ colors and having at most $m$ intervals of each color (van Bevern et al., 2015), where the task is to find a maximum-weight independent set of intervals with mutually distinct colors.

Colorful IndePendent Set is fixed-parameter tractable parameterized by the number of colors in the solution on interval (and even on chordal) graphs (van Bevern et al., 2015; Bentert et al., 2017), which implies that $P\left|M_{j}, d_{j}-r_{j}=p_{j}\right| \sum w_{j} U_{j}$ is fixed-parameter tractable parameterized by the number of jobs that can be scheduled in an optimal solution. To answer Open Problem 8, one could try to show that Colorful InDEPENDENT SET in interval graphs is fixed-parameter tractable parameterized by the maximum number of intervals of any color.

\subsection{Allowed preemption}

The three problems $P\left|\mathrm{pmtn}, r_{j}\right| \Sigma C_{j}, P|\operatorname{pmtn}| \Sigma U_{j}$, and $P|\mathrm{pmtn}| \Sigma T_{j}$ are all NP-hard (Du et al., 1990; Lawler, 1983; Kravchenko and Werner, 2013), yet the special cases with equal processing times are polynomial-time solvable (Baptiste et al., 2004, 2007; Kravchenko and Werner, 2011).

Open Problem 9. Are the above problems fixed-parameter tractable parameterized by the number $\bar{p}$ of distinct processing times?

Kravchenko and Werner (2011) survey related problems; as of now, the complexity status of $P 2\left|\mathrm{pmtn}, p_{j}=p\right| \Sigma w_{j} T_{j}$ for constant $p$ is open. Parameterized hardness might serve as an intermediate step towards settling it.

Open Problem 10. Is P2|pmtn, $p_{j}=p \mid \sum w_{j} T_{j}$ W[1]-hard parameterized by the processing time $p$ of all jobs?

\section{Shop scheduling}

We now consider the open shop, job shop, and flow shop scheduling problems. Section 5.1 considers the makespan objective, Section 5.2 considers fixed-parameter approximation schemes, Section 5.3 takes into account setup times, and, finally, Section 5.4 considers variants of maximizing throughput.

\subsection{Makespan}

Kononov et al. (2012) give a computational complexity dichotomy of $J \| C_{\max }$ and $O \| C_{\max }$ into polynomial-time solvable cases and NP-hard cases depending on the maximum processing time $p_{\max }$ of operations, the maximum number $n_{\max }$ of operations per job, and an upper bound on the makespan $C_{\max }$. All problems are NP-hard even if all of the listed parameters are simultaneously bounded from above by 4 (Kononov et al., 2012; Williamson et al., 1997), which fully settles the parameterized complexity of $O \| C_{\max }$ and $J \| C_{\max }$ with respect to these parameters.

However, in all hardness reductions of Kononov et al. (2012), the number of machines is necessarily unbounded: when bounding both the number $m$ of machines and the makespan $C_{\max }$, then shop scheduling problems are trivial, since the overall number of operations in the input is at most $m \cdot C_{\max }$. This makes the parameters $p_{\max }$ and $n_{\max }$ interesting for fixed-parameter algorithms for shop scheduling problems with a fixed number of machines (or with the number of machines as an additional parameter).

For example, both $F 3 \| C_{\max }$ and $O 3 \| C_{\max }$ are strongly NPhard (Gonzalez and Sahni, 1976; Garey et al., 1976), yet $O m \| C_{\max }$ is fixed-parameter tractable parameterized by maximum processing time $p_{\max }$ : if the maximum machine load is $\ell_{\max } \geq m^{2} p_{\max }$, then the makespan of the instance is $\ell_{\max }$ (Sevast'janov, 1995). Otherwise, there are at most $\ell_{\max }<m^{2} p_{\max }$ jobs and the instance can be solved using brute force. For $F 3 \| C_{\text {max }}$, the analogous question is open:

Open Problem 11. Is $F 3 \| C_{\max }$ fixed-parameter tractable parameterized by the maximum processing time $p_{\max }$ ?

This question is likewise interesting for $F 3 \mid$ no-wait $\mid C_{\max }$, where "no-wait" means that each operation of a job has to start immediately after completion of the preceding operation of the same 
job. The NP-hardness of $F 3 \mid$ no-wait| $C_{\max }$ was a long-standing open question (Röck, 1984).

Notably, for the three-machine job shop scheduling problem, the above question has a negative answer: even the special case $J 3\left|p_{i j}=1\right| C_{\max }$ is NP-hard (Lenstra and Rinnooy Kan, 1979).

Open Problem 12. Is $J 3 \| C_{\max }$ fixed-parameter tractable parameterized by the maximum processing time $p_{\max }$ and the maximum number $n_{\max }$ of operations per job?

\subsection{Makespan approximation}

Job and open shop scheduling problems are often APX-hard (Williamson et al., 1997) but also NP-hard for a constant number of machines (Gonzalez and Sahni, 1976; Garey et al., 1976; Lenstra and Rinnooy Kan, 1979) and many other constant parameters (Kononov et al., 2012). Thus, they are amenable neither to approximation schemes nor fixed-parameter algorithms.

However, while having a constant number of machines does not help to get polynomial-time algorithms for these problems, it helps tremendously in getting PTASes (Sevastianov and Woeginger, 1998; Hall, 1998; Jansen et al., 2003). Remarkably, these are not simply PTASes for a fixed number of machines running in time, say $n^{O(m / \varepsilon)}$ : In terms of Marx (2008), they are actually fixed-parameter tractable approximation schemes (FPT-AS) for the parameters $m$ and $\varepsilon$, running in time $f(m, \varepsilon) \cdot \operatorname{poly}(n)$. While not necessarily being a PTAS, an FPT-AS using the number $m$ of machines as parameter and running in $O\left(2^{m / \varepsilon} \cdot n\right)$ time might be practically more valuable than a PTAS running in $O\left(n^{1 / \varepsilon}\right)$ time or even an FPTAS running in $O\left((n+1 / \varepsilon)^{3}\right)$ time.

Sevastianov and Woeginger $(1998)$ compute a $(1+\varepsilon)$-approximation for $O \| C_{\max }$ in $f(m, \varepsilon)+O(n \log n)$ time, Hall (1998) computes a $(1+\varepsilon)$-approximation for $F \| C_{\max }$ in $f(m, \varepsilon)+O\left(n^{3.5}\right)$ time. Yet for $J \| C_{\max }$, Jansen et al. (2003) need an additional parameter: they compute a $(1+\varepsilon)$-approximation for $J \| C_{\max }$ in $f\left(m, n_{\max }, \varepsilon\right)+O(n)$ time, where $n_{\max }$ is the maximum number of operations of a job.

Open Problem 13. Is there an algorithm that yields $a(1+\varepsilon)$ approximation for $J \| C_{\max }$ in $f(m, \varepsilon) \cdot \operatorname{poly}(n)$ time?

Giving a negative answer to this question seems challenging: the obvious approach would be proving that $J \| C_{\max }$ is $\mathrm{W}[1]-$ hard parameterized by $C_{\max }+m$. However, as discussed in Section 5.1, $J \| C_{\max }$ is trivially fixed-parameter tractable parameterized by $C_{\max }+m$, so that this approach is inapplicable.

\subsection{Makespan with sequence-dependent setup times}

In the following, we consider a variant $O\left|p_{i j}=1, \mathrm{ST}_{\mathrm{sd}, b}\right| C_{\max }$ of open shop with unit processing times where the jobs are partitioned into $g$ batches and machines require a sequencedependent batch setup time $s_{p q} \in \mathbb{N}$ when switching from jobs of batch $p$ to jobs of batch $q$.

The case with unit processing times models applications where large batches of items have to be processed and processing individual items in each batch takes significantly less time than setting up the machine for the batch. Alternatively one can interpret this problem as an open shop problem where machines or experts have to travel between jobs in different locations in order to process them (Averbakh et al., 2006).

As a generalization of the Traveling SALESPERson problem, $O\left|p_{i j}=1, \mathrm{ST}_{\mathrm{sd}, b}\right| C_{\max }$ is NP-hard already for one machine. As shown by van Bevern and Pyatkin (2016), the problem is solvable in $O\left(2^{g} g^{2}+m n\right)$ time if each batch contains more jobs than there are machines. For the case where batches may contain less jobs, they showed that the problem is solvable in $O(n \log n)$ time if both the number $m$ of machines and the number $g$ of batches are constants.

Open Problem 14. Is $O\left|p_{i j}=1, S T_{s d, b}\right| C_{\max }$ fixed-parameter tractable parameterized by the number $g$ of batches?

We point out that, for this problem, it would be desirable to design a fixed-parameter algorithm that works also for the highmultiplicity encoding, which compactly encodes the number of jobs in each batch in binary. The fixed-parameter algorithms of van Bevern and Pyatkin (2016) also work for the high-multiplicity encoding as long as they only need to compute the minimum makespan.

\subsection{Throughput or weighted number of tardy jobs}

Since checking for a schedule with makespan $L$ is equivalent to checking whether all jobs can meet a due date of $L$, all NPhardness results from $J \| \Sigma C_{j}$ and $O \| \Sigma C_{j}$ in Section 5.1 carry over to $J \| \Sigma U_{j}$ and $O \| \Sigma U_{j}$. In fact, these throughput maximization variants turn out to be even harder: now, even the special cases $J 2\left|p_{i j}=1\right| \Sigma w_{j} U_{j}$ and $J 2\left|p_{i j}=1, r_{j}\right| \Sigma U_{j}$ on two machines are NPhard (Kravchenko, 2000b; Timkovsky, 1998).

The situation is more relaxed for open shop scheduling: $O\left|p_{i j}=1, r_{j}\right| \Sigma U_{j}$ is NP-hard for an unbounded number of machines (Kravchenko, 2000a), whereas $O\left|p_{i j}=1\right| \sum w_{j} U_{j}$ is even fixed-parameter tractable parameterized by the number $m$ of machines (Brucker et al., 1993b). For $O m\left|p_{i j}=1, r_{j}\right| \sum w_{j} U_{j}$, we only know polynomial-time solvability for a constant number of machines (Baptiste, 2003).

Open Problem 15. Is $O\left|p_{i j}=1, r_{j}\right| \sum w_{j} U_{j}$ fixed-parameter tractable parameterized by the number of machines?

It is known that the special case $O\left|p_{i j}=1, r_{j}\right| C_{\max }$ is polynomialtime solvable and that $O\left|p_{i j}=1, r_{j}\right| \Sigma w_{j} U_{j}$ is equivalent to the problem $P\left|p_{j}=m, \operatorname{pmtn}_{\mathbb{Z}}, r_{j}\right| \Sigma w_{j} U_{j}$ of scheduling jobs with processing time $m$ on $m$ parallel identical machines with release dates and preemption allowed at integer times (Brucker et al., 1993a).

Acknowledgements. Matthias Mnich is supported by DFG grant MN 59/4-1 and ERC Starting Grant 306465 (BeyondWorstCase); he expresses his gratitude towards Alexander Grigoriev for enlightening discussions and many helpful comments.

René van Bevern is supported by the Russian Foundation for Basic Research, project 16-31-60007 mol_a_dk, and by the Ministry of Science and Education of the Russian Federation under the 5-100 Excellence Program; he thanks Gerhard J. Woeginger for pointing out Open Problem 11.

Both authors thank Martin Koutecký for pointing out that $P \| C_{\max }$ is fixed-parameter tractable for the number of distinct 
processing times when those are encoded in unary and Gerhard $\mathrm{J}$. Woeginger for pointing out that $O 3 \| C_{\max }$ is fixed-parameter tractable when parameterized by the maximum processing time. They also thank the reviewers for their detailed and helpful remarks which lead to an improved presentation.

\section{References}

Ali Allahverdi, C.T. Ng, T.C.E. Cheng, and Mikhail Y. Kovalyov. A survey of scheduling problems with setup times or costs. European Journal of Operational Research, 187(3):985-1032, 2008. doi:10.1016/j.ejor.2006.06.060.

Noga Alon, Yossi Azar, Gerhard J. Woeginger, and Tal Yadid. Approximation schemes for scheduling on parallel machines. Journal of Scheduling, 1(1):55-66, 1998. doi:10.1002/(SICI)1099-1425(199806)1:1<55::AIDJOS2>3.3.CO;2-A.

Christoph Ambühl and Monaldo Mastrolilli. Single machine precedence constrained scheduling is a vertex cover problem. Algorithmica, 53(4):488-503, 2009. doi:10.1007/s00453-008-9251-6.

Esther M. Arkin and Ellen B. Silverberg. Scheduling jobs with fixed start and end times. Discrete Applied Mathematics, 18(1):1-8, 1987. doi:10.1016/0166218X(87)90037-0.

Igor Averbakh, Oded Berman, and Ilya Chernykh. The routing open-shop problem on a network: complexity and approximation. European Journal of Operational Research, 173(2):531-539, 2006. doi:10.1016/j.ejor.2005.01.034.

Philippe Baptiste. On minimizing the weighted number of late jobs in unit execution time open-shops. European Journal of Operational Research, 149 (2):344-354, 2003. doi:10.1016/S0377-2217(02)00759-2.

Philippe Baptiste, Peter Brucker, Sigrid Knust, and Vadim G. Timkovsky. Ten notes on equal-processing-time scheduling: at the frontiers of solvability in polynomial time. 4OR. A Quarterly Journal of Operations Research, 2(2): 111-127, 2004. doi:10.1007/s10288-003-0024-4.

Philippe Baptiste, Peter Brucker, Marek Chrobak, Christoph Dürr, Svetlana A Kravchenko, and Francis Sourd. The complexity of mean flow time scheduling problems with release times. Journal of Scheduling, 10(2):139-146, 2007. doi:10.1007/s10951-006-0006-4.

Matthias Bentert, René van Bevern, and Rolf Niedermeier. (wireless) scheduling, graph classes, and $c$-colorable subgraphs, 2017. Preprint. https : //arxiv . org/abs/1712.06481.

René van Bevern and Artem V. Pyatkin. Completing partial schedules for Open Shop with unit processing times and routing. In Proceedings of the 11th International Computer Science Symposium in Russia (CSR'16), volume 9691 of Lecture Notes Comput. Sci., pages 73-87, 2016. doi:10.1007/978-3319-34171-2_6. (improved version on arXiv:1603.01191).

René van Bevern, Matthias Mnich, Rolf Niedermeier, and Mathias Weller. Interval scheduling and colorful independent sets. Journal of Scheduling, 18 (5):449-469, 2015. doi:10.1007/s10951-014-0398-5.

René van Bevern, Robert Bredereck, Laurent Bulteau, Christian Komusiewicz, Nimrod Talmon, and Gerhard J. Woeginger. Precedence-constrained scheduling problems parameterized by partial order width. In Proceedings of the 9th International Conference on Discrete Optimization and Operations Research (DOOR'16), volume 9869 of Lecture Notes Comput. Sci., pages 105-120, 2016. doi:10.1007/978-3-319-44914-2_9.

René van Bevern, Christian Komusiewicz, and Manuel Sorge. A parameterized approximation algorithm for the mixed and windy capacitated arc routing problem: Theory and experiments. Networks, 70(3):262-278, 2017a. doi:10.1002/net.21742.

René van Bevern, Rolf Niedermeier, and Ondřej Suchý. A parameterized complexity view on non-preemptively scheduling interval-constrained jobs: few machines, small looseness, and small slack. Journal of Scheduling, 20 (3):255-265, 2017b. doi:10.1007/s10951-016-0478-9.

Jean-Charles Billaut and Francis Sourd. Single machine scheduling with forbidden start times. 4OR. A Quarterly Journal of Operations Research, 7(1): 37-50, 2009. doi:10.1007/s10288-007-0061-5.

Hans L. Bodlaender and Michael R. Fellows. W[2]-hardness of precedence constrained $K$-processor scheduling. Operations Research Letters, 18(2): 93-97, 1995. doi:10.1016/0167-6377(95)00031-9.

Hans L. Bodlaender, Bart M. P. Jansen, and Stefan Kratsch. Kernelization lower bounds by cross-composition. SIAM Journal on Discrete Mathematics, 28 (1):277-305, 2014. doi:10.1137/120880240.
Nadia Brauner, Yves Crama, Alexander Grigoriev, and Joris van de Klundert. A framework for the complexity of high-multiplicity scheduling problems. Journal of Combinatorial Optimization, 9(3):313-323, 2005. doi:10.1007/s10878005-1414-7.

Peter Brucker and Svetlana A. Kravchenko. Preemption can make parallel machine scheduling problems hard. Technical Report 211, Universität Osnabrück, Fachbereich Mathematik/Informatik, 1999.

Peter Brucker, Bernd Jurisch, and Marion Jurisch. Open shop problems with unit time operations. Zeitschrift für Operations Research, 37(1):59-73, 1993a. doi:10.1007/BF01415528.

Peter Brucker, Bernd Jurisch, Thomas Tautenhahn, and Frank Werner. Scheduling unit time open shops to minimize the weighted number of late jobs. Operations Research Letters, 14(5):245-250, 1993b. doi:10.1016/01676377(93)90088-X.

Marco Cesati and Luca Trevisan. On the efficiency of polynomial time approximation schemes. Information Processing Letters, 64(4):165-171, 1997. doi:10.1016/S0020-0190(97)00164-6.

Lin Chen, Dániel Marx, Deshi Ye, and Guochuan Zhang. Parameterized and approximation results for scheduling with a low rank processing time matrix. In Proceedings of the 34th Symposium on Theoretical Aspects of Computer Science (STACS'17), volume 66 of Leibniz Int. Proc. Informatics, pages 22:1-22:14, 2017. doi:10.4230/LIPIcs.STACS.2017.22.

Mark Cieliebak, Thomas Erlebach, Fabian Hennecke, Birgitta Weber, and Peter Widmayer. Scheduling with release times and deadlines on a minimum number of machines. In Exploring New Frontiers of Theoretical Informatics, volume 155 of IFIP International Federation for Information Processing, pages 209-222. 2004. doi:10.1007/1-4020-8141-3_18.

Marek Cygan, Fedor V. Fomin, Łukasz Kowalik, Daniel Lokshtanov, Dániel Marx, Marcin Pilipczuk, Michał Pilipczuk, and Saket Saurabh. Parameterized algorithms. Springer, Cham, 2015. doi:10.1007/978-3-319-21275-3.

Jianzhong Du, Joseph Y.-T. Leung, and Gilbert H. Young. Minimizing mean flow time with release time constraint. Theoretical Computer Science, 75(3) 347-355, 1990. doi:10.1016/0304-3975(90)90100-V.

Michael Etscheid, Stefan Kratsch, Matthias Mnich, and Heiko Röglin. Polynomial kernels for weighted problems. Journal of Computer and System Sciences, 84:1-10, 2017. doi:10.1016/j.jcss.2016.06.004.

Michael R. Fellows and Catherine McCartin. On the parametric complexity of schedules to minimize tardy tasks. Theoretical Computer Science, 298(2): 317-324, 2003. doi:10.1016/S0304-3975(02)00811-3.

Michael R. Fellows, Bart M. P. Jansen, and Frances A. Rosamond. Towards fully multivariate algorithmics: Parameter ecology and the deconstruction of computational complexity. European Journal of Combinatorics, 34(3) 541-566, 2013. doi:10.1016/j.ejc.2012.04.008.

Jörg Flum and Martin Grohe. Parameterized Complexity Theory. Springer, 2006. doi:10.1007/3-540-29953-X

Michaël Gabay, Christophe Rapine, and Nadia Brauner. High-multiplicity scheduling on one machine with forbidden start and completion times. Journal of Scheduling, 19(5):609-616, 2016. doi:10.1007/s10951-016-0475-z.

Michael R. Garey and David S. Johnson. Computers and intractability. W. H. Freeman and Co., San Francisco, Calif., 1979. A guide to the theory of NP-completeness, A Series of Books in the Mathematical Sciences.

Michael R. Garey, David S. Johnson, and Ravi Sethi. The complexity of flowshop and jobshop scheduling. Mathematics of Operations Research, 1(2):117-129, 1976. doi:10.1287/moor.1.2.117.

Michel X. Goemans and Thomas Rothvoß. Polynomiality for bin packing with a constant number of item types. In Proceedings of the 25th Annual ACMSIAM Symposium on Discrete Algorithms (SODA'14), pages 830-839, 2014. doi:10.1137/1.9781611973402.61.

Teofilo Gonzalez and Sartaj Sahni. Open shop scheduling to minimize finish time. Journal of the Association for Computing Machinery, 23(4):665-679, 1976. doi: $10.1145 / 321978.321985$.

Ronald L. Graham, Eugene L. Lawler, Jan K. Lenstra, and Alexander H. G. Rinnooy Kan. Optimization and approximation in deterministic sequencing and scheduling: a survey. Annals of Discrete Mathematics, 5:287-326, 1979. Discrete optimization (Proc. Adv. Res. Inst. Discrete Optimization and Systems Appl., Banff, Alta., 1977), II.

Elisabeth Günther, Felix G. König, and Nicole Megow. Scheduling and packing malleable and parallel tasks with precedence constraints of bounded width. Journal of Combinatorial Optimization, 27(1):164-181, 2014. doi:10.1007/s10878-012-9498-3.

Jiong Guo and Rolf Niedermeier. Invitation to data reduction and problem kernel- 
ization. SIGACT News, 38(1):31-45, 2007. doi:10.1145/1233481.1233493.

Leslie A. Hall. Approximability of flow shop scheduling. Mathematical Programming, 82(1-2, Ser. B):175-190, 1998. doi:10.1007/BF01585870.

Magnús M. Halldórsson and Ragnar K. Karlsson. Strip graphs: recognition and scheduling. In Proceedings of the 32nd International Workshop on GraphTheoretic Concepts in Computer Science (WG'06), volume 4271 of Lecture Notes Comput. Sci., pages 137-146. 2006. doi:10.1007/11917496_13.

Danny Hermelin, Judith-Madeleine Kubitza, Dvir Shabtay, Nimrod Talmon, and Gerhard Woeginger. Scheduling two competing agents when one agent has significantly fewer jobs. In Proceedings of the 10th International Symposium on Parameterized and Exact Computation (IPEC'15), volume 43 of Leibniz Int. Proc. Informatics, pages 55-65. 2015. doi:10.4230/LIPIcs.IPEC.2015.55.

Danny Hermelin, Dvir Shabtay, and Nimrod Talmon. On the parameterized tractability of the just-in-time flow-shop scheduling problem, 2017. Preprint https://arxiv.org/abs/1709.04169.

Danny Hermelin, Shlomo Karhi, Michael Pinedo, and Dvir Shabtay. New algorithms for minimizing the weighted number of tardy jobs on a single machine. Annals of Operations Research, 2018. doi:10.1007/s10479-0182852-9. In press.

Klaus Jansen and Kim-Manuel Klein. About the structure of the integer cone and its application to bin packing. In Proceedings of the 28th Annual ACM-SIAM Symposium on Discrete Algorithms (SODA'17), pages 1571-1581, 2017. doi:10.1137/1.9781611974782.103.

Klaus Jansen, Roberto Solis-Oba, and Maxim Sviridenko. Makespan minimization in job shops: a linear time approximation scheme. SIAM Journal on Discrete Mathematics, 16(2):288-300, 2003. doi:10.1137/S0895480199363908.

Klaus Jansen, Marten Maack, and Roberto Solis-Oba. Structural parameters for scheduling with assignment restrictions. In Proceedings of the 10th International Conference on Algorithms and Complexity (CIAC'17), volume 10236 of Lecture Notes Comput. Sci., pages 357-368, 2017. doi:10.1007/9783-319-57586-5 30.

Richard M. Karp. Reducibility among combinatorial problems. In Complexity of computer computations (Proc. Sympos., IBM Thomas J. Watson Res. Center, Yorktown Heights, N.Y., 1972), pages 85-103, 1972.

Dušan Knop and Martin Koutecký. Scheduling meets $n$-fold integer programming. Journal of Scheduling, 2017. doi:10.1007/s10951-017-0550-0.

Dušan Knop, Martin Koutecký, and Matthias Mnich. Combinatorial $n$-fold integer programming and applications. In Proceedings of the 25th Annual European Symposium on Algorithms (ESA '17), volume 87 of Leibniz Int. Proc. Informatics, pages 54:1-54:14, 2017. doi:10.4230/LIPIcs.ESA.2017.54.

Christian Komusiewicz and Rolf Niedermeier. New races in parameterized algorithmics. In Proceedings of the 37th International Symposium on Mathematical Foundations of Computer Science (MFCS'12), volume 7464 of Lecture Notes Comput. Sci., pages 19-30, 2012. doi:10.1007/978-3-64232589-2_2.

Alexander Kononov, Sergey Sevastyanov, and Maxim Sviridenko. A complete 4-parametric complexity classification of short shop scheduling problems. Journal of Scheduling, 15(4):427-446, 2012. doi:10.1007/s10951-011-0243$\mathrm{z}$.

Stefan Kratsch. Recent developments in kernelization: A survey. Bulletin of the EATCS, 113, 2014. http://eatcs.org/beatcs/index.php/beatcs/ article/view/285.

Stefan Kratsch and Magnus Wahlström. Representative sets and irrelevant vertices: New tools for kernelization. In Proceedings of the 53rd Annual Symposium on Foundations of Computer Science (FOCS'12), pages 450-459, 2012. doi:10.1109/FOCS.2012.46.

Svetlana A. Kravchenko. On the complexity of minimizing the number of late jobs in unit time open shop. Discrete Applied Mathematics, 100(1-2): 127-132, 2000a. doi:10.1016/S0166-218X(99)00202-4.

Svetlana A. Kravchenko. Minimizing the number of late jobs for the twomachine unit-time job-shop scheduling problem. Discrete Applied Mathematics, 98(3):209-217, 2000b. doi:10.1016/S0166-218X(99)00165-1.

Svetlana A. Kravchenko and Frank Werner. Parallel machine problems with equal processing times: a survey. Journal of Scheduling, 14(5):435-444, 2011. doi:10.1007/s10951-011-0231-3.

Svetlana A. Kravchenko and Frank Werner. Erratum to: Minimizing total tardiness on parallel machines with preemptions. Journal of Scheduling, 16 (4):439-441, 2013. doi:10.1007/s10951-013-0313-5.

Eugene L. Lawler. A “pseudopolynomial” algorithm for sequencing jobs to minimize total tardiness. In Studies in Integer Programming, volume 1 of Annals of Discrete Mathematics, Supplement C, pages 331-342. Elsevier, 1977. doi:10.1016/S0167-5060(08)70742-8.

Eugene L. Lawler. Recent results in the theory of machine scheduling. In Mathematical Programming: The State of the Art (Bonn, 1982), pages 202234. Springer, 1983. doi:10.1007/978-3-642-68874-4_9.

Eugene L. Lawler and J. Michael Moore. A functional equation and its application to resource allocation and sequencing problems. Management Science, 16(1):77-84, 1969. doi:10.1287/mnsc.16.1.77.

Jan K. Lenstra and Alexander H. G. Rinnooy Kan. Computational complexity of discrete optimization problems. Annals of Discrete Mathematics, 4:121-140, 1979. doi:10.1016/S0167-5060(08)70821-5.

Jan K. Lenstra, Alexander H. G. Rinnooy Kan, and Peter Brucker. Complexity of machine scheduling problems. In Studies in Integer Programming, volume 1 of Annals of Discrete Mathematics, Supplement C, pages 343-362. Elsevier, 1977. doi:10.1016/S0167-5060(08)70743-X.

Dániel Marx. Parameterized complexity and approximation algorithms. The Computer Journal, 51(1):60, 2008. doi:10.1093/comjn1/bxm048.

Dániel Marx and Ildikó Schlotter. Stable assignment with couples: parameterized complexity and local search. Discrete Optimization, 8(1):25-40, 2011. doi:10.1016/j.disopt.2010.07.004.

Neeldhara Misra, Venkatesh Raman, and Saket Saurabh. Lower bounds on kernelization. Discrete Optimization, 8(1):110-128, 2011. doi:10.1016/j.disopt.2010.10.001.

Matthias Mnich and Andreas Wiese. Scheduling and fixed-parameter tractability. Mathematical Programming, Series B, 154(1-2):533-562, 2015. doi:10.1007/s10107-014-0830-9.

Kazuo Nakajima and S. Louis Hakimi. Complexity results for scheduling tasks with discrete starting times. Journal of Algorithms, 3(4):344-361, 1982. doi:10.1016/0196-6774(82)90030-X.

Rolf Niedermeier. Invitation to Fixed-Parameter Algorithms, volume 31 of Oxford Lecture Series in Mathematics and Its Applications. Oxford University Press, 2006. doi:10.1093/acprof:oso/9780198566076.001.0001.

Rolf Niedermeier. Reflections on multivariate algorithmics and problem parameterization. In Proceedings of the 27th International Symposium on Theoretical Aspects of Computer Science (STACS'10), volume 5 of Leibniz Int. Proc. Informatics, pages 17-32. Schloss Dagstuhl-Leibniz-Zentrum für Informatik, 2010. doi:10.4230/LIPIcs.STACS.2010.2495.

Christophe Rapine and Nadia Brauner. A polynomial time algorithm for makespan minimization on one machine with forbidden start and completion times. Discrete Optimization, 10(4):241-250, 2013. doi:10.1016/j.disopt.2013.07.003.

Hans Röck. The three-machine no-wait flow shop is NP-complete. Journal of the Association for Computing Machinery, 31(2):336-345, 1984. doi: $10.1145 / 62.65$.

Petra Schuurman and Gerhard J. Woeginger. Polynomial time approximation algorithms for machine scheduling: ten open problems. Journal of Scheduling, 2(5):203-213, 1999. doi:10.1002/(SICI)10991425(199909/10)2:5<203::AID-JOS26>3.3.CO;2-X.

Sergey V. Sevastianov and Gerhard J. Woeginger. Makespan minimization in open shops: a polynomial time approximation scheme. Mathematical Programming, 82(1-2, Ser. B):191-198, 1998. doi:10.1007/BF01585871.

Sergey Sevast'janov. Vector summation in banach space and polynomial algorithms for flow shops and open shops. Mathematics of Operations Research, 20(1):90-103, 1995. doi:10.1287/moor.20.1.90.

Jiří Sgall. Open problems in throughput scheduling. In Proceedings of the 20th Annual European Symposium on Algorithms (ESA'12), volume 7501 of Lecture Notes Comput. Sci., pages 2-11, 2012. doi:10.1007/978-3-64233090-2_2.

Shao Chin Sung and Milan Vlach. Maximizing weighted number of just-in-time jobs on unrelated parallel machines. Journal of Scheduling, 8(5):453-460, 2005. doi:10.1007/s10951-005-2863-7.

Vadim G. Timkovsky. Is a unit-time job shop not easier than identical parallel machines? Discrete Applied Mathematics, 85(2):149-162, 1998. doi:10.1016/S0166-218X(98)00032-8.

David P. Williamson, Leslie A. Hall, Jan A. Hoogeveen, Cor A. J. Hurkens, Jan K. Lenstra, Sergey V. Sevast'janov, and David B. Shmoys. Short shop schedules. Operations Research, 45(2):288-294, 1997. doi:10.1287/opre.45.2.288. 